\title{
Pascal à Mirefleurs ? Les dessins de la maison de Domat
}

Ezio Ardvini et Yves Morvan

\section{(2) OpenEdition}

Journals

Édition électronique

URL : http://journals.openedition.org/ccibp/427

DOI : $10.4000 /$ ccibp. 427

ISSN : 2493-7460

Éditeur

Centre international Blaise Pascal

Édition imprimée

Date de publication : 16 octobre 1984

Pagination : 6-17

ISSN : 0249-6674

\section{Référence électronique}

Ezio Ardvini et Yves Morvan, "Pascal à Mirefleurs ? Les dessins de la maison de Domat », Courrier du Centre international Blaise Pascal [En ligne], 6| 1984, mis en ligne le 27 novembre 2015, consulté le 02 mai 2019. URL : http://journals.openedition.org/ccibp/427 ; DOI : 10.4000/ccibp.427

Ce document a été généré automatiquement le 2 mai 2019.

Centre international Blaise Pascal 


\title{
Pascal à Mirefleurs? Les dessins de la maison de Domat
}

\author{
Ezio Ardvini et Yves Morvan
}

\section{Situation géographique et historique}

1 La commune de Mirefleurs est située sur la rive droite de l'Allier, à dix-huit kilomètres au Sud de Clermont-Ferrand. Superficie : 904 ha ; 1600 habitants ; longitude Est : $3^{\circ} 10^{\prime} 27$; latitude Nord : $45^{\circ} 41$ ' 40 . La rivière Allier la borde, coulant du Sud au Nord-Ouest ; le puy de Saint-André (678 $\mathrm{m}$ ) ferme son horizon au Nord, et le puy de Saint-Romain $(779 \mathrm{~m})$ au Sud. On peut dire sans conteste que le village est charmant. (fig. 1). 
Figure 1 Mirefleurs vue générale prise vers le Nord-Ouest.

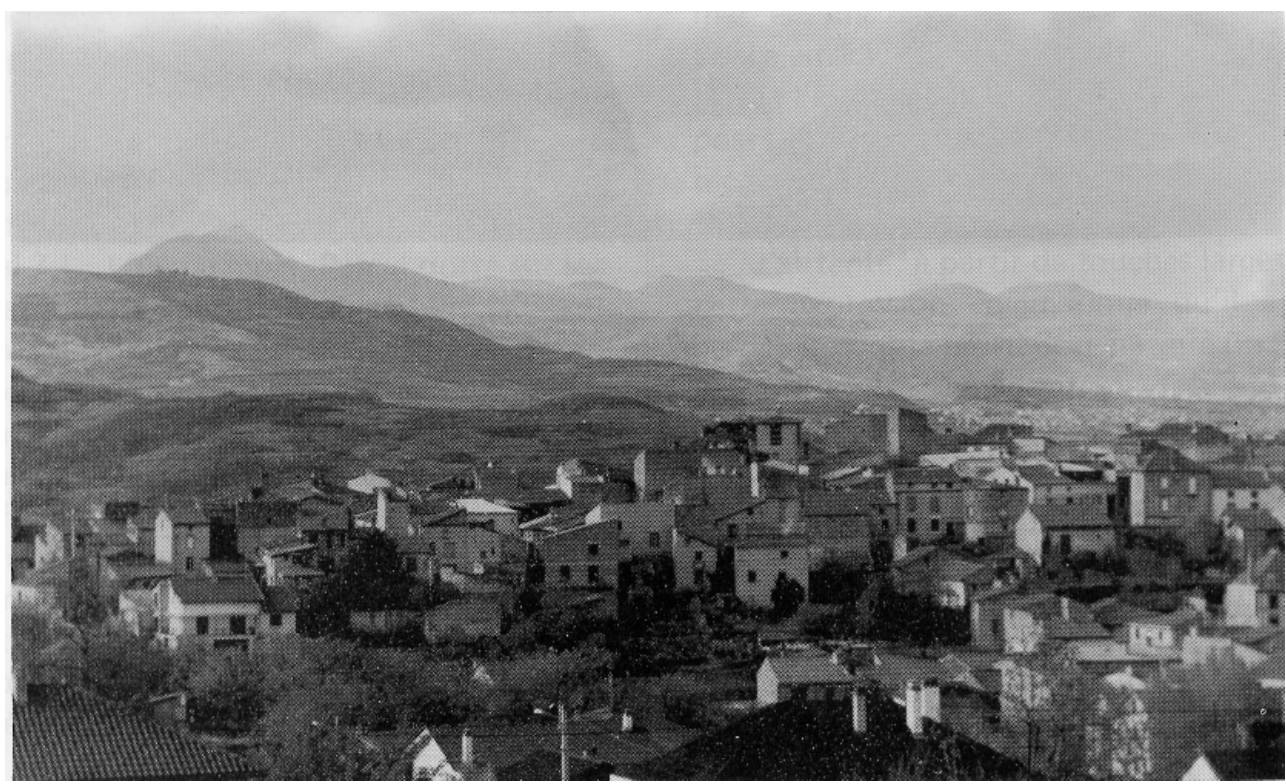

La chaîne des Dômes dans le fond

2 Le nom celtique du lieu est oublié. Le village primitif, bâti à $420 \mathrm{~m}$ d'altitude sur un promontoire accolé au massif du puy de Saint-André, s'appelait Castrum novum supra Ligerim, ou Châteauneuf-sur-Allier, et fut fortifié vers la fin du XII ${ }^{e}$ siècle. En 1509 on le nomme encore "Mirefleurs ci-devant Châteauneuf». En effet le changement de nom n'eut lieu qu'en 1470. On raconte que cette année le comte d'Auvergne, Bertrand VII de la Tour, qui résidait non loin de là à Vic-le-Comte, se trouvant à la chasse dans cette belle région dit aux chevaliers qui l'accompagnaient et qui admiraient le site: "Mirez ces fleurs, Messires». Ensuite de quoi le roi Louis XI, pour lui faire plaisir, ordonna le changement de nom par une lettre patente conservée à la bibliothèque de l'Arsenal à Paris, datée d'Amboise le 15 décembre 1470.

3 Au temps de la Ligue, Mirefleurs, qui était pour le roi, fut inquiété en 1589 par les armées du comte de Randan, et pris par celles du duc de Nemours, en juin-juillet 1591. Le secours envoyé par M. de Florat, sénéchal de Clermont, arriva lorsque les assaillants étaient déjà dans la place. En octobre 1591 les armées de la Ligue consentirent à se retirer moyennant une grosse rançon payée par les habitants de Vic-le-Comte.

Aujourd'hui encore on peut se faire une idée du tracé des remparts et situer quelques tours. Une lettre de Charles VIII, datée du 19 octobre 1591, dit que c'est « une bonne ville close à côté d'une haute montagne où il y a grand terrier et roc ». Cette lettre ordonne au bailli de Montferrand de contraindre les habitants à réparer le très dangereux chemin d'Esparron situé sur le bord de la rivière d'Allier. En 1653 les portes de l'enceinte qui entourait le bourg attenant au château existaient encore avec leurs serrures. Elles avaient nom : porte du Godel, en direction de l'avantposte de Dieu-y-soit, sur l'Allier; porte de l'Espinet, à l'Est; et le Pourchey (le porche) pour la porte principale vers le Nord ; toutes permettant l'accès des voitures. Pour les piétons une petite porte, dite du Guichet ou du Saint-Esprit, débouchait près de la chapelle du château, à l'emplacement de l'actuelle église paroissiale. 
5 La cure dépendait de La Chaise-Dieu qui y avait envoyé un petit prieuré. Vers 1560, Gilbert du Lac, capitaine du château de Mirefleurs et de Busséol, lui fait un legs de cent livres tournois, aux bons soins de la reine de France, Catherine de Médicis, comtesse d'Auvergne. L'abbé de la ChaiseDieu avait la nomination du curé jusqu'en 1789 et le patron de la paroisse était saint Genès.

\section{La maison de Domat et la pièce aux dessins}

6 Située au cœur du village de Mirefleurs, la maison dite « de Domat» (fig. 4) appartient actuellement à Monsieur Henri Desfeuilles.

7 Jean DOMAT - né à Clermont, le 30 novembre 1625, mort à Paris le 14 mars 1696 - était ce jurisconsulte auquel Pascal, en mourant, confia ses papiers, ce qui dit assez bien quels liens d'amitié liaient les deux hommes et justifie l'intérêt porté à cette demeure par les historiens de Pascal.

Cette maison, dont l'origine pourrait remonter au $\mathrm{XV}^{\mathrm{e}}$ siècle en raison de cheminées gothiques encore en place et d'un faux appareil peint en claveaux colorés à la manière médiévale, s'est enrichie, au cours des siècles, d'ajouts qui ne semblent pas l'avoir fondamentalement transformée. La grande fenêtre renaissance de la façade, au deuxième étage, qui a dû remplacer une ouverture plus petite, et la porte d'entrée de style Louis XV sont la marque apparente de ces âges successifs.

Fig. 2a : Médaillon représentant Pascal.

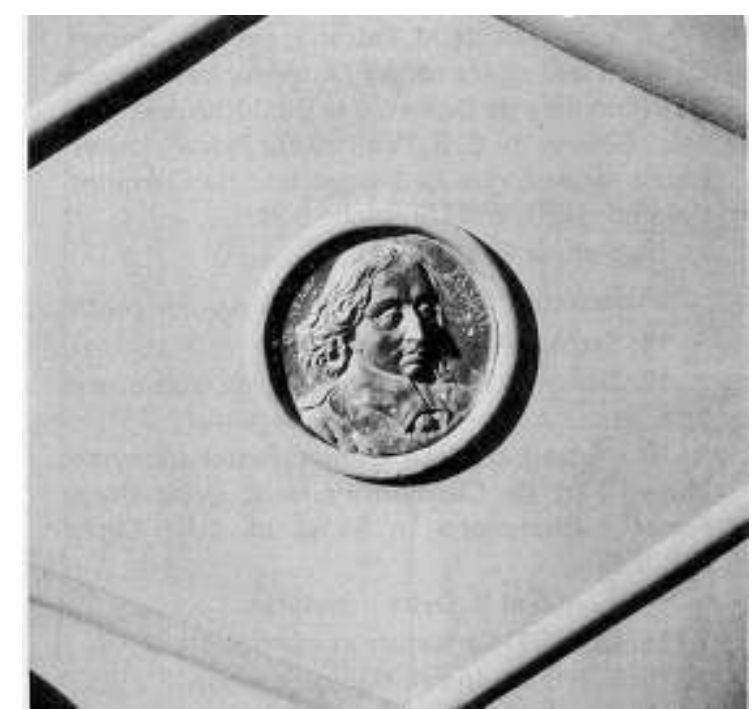

Mirefleurs, quartier du Pavé. Portail de la maison Roubille. De la fonderie, Barbier-Daubrée, 1848.

Photo Ezio Arduini.

Cette grande maison sert de local d'hébergement à un groupe d'archéologues anglais qui l'occupe à la belle saison et y entrepose le produit de ses fouilles.

Elle comporte un rez-de-chaussée et deux étages desservis par un escalier à vis formant tour. Au second étage un escalier de bois permet d'accéder à une pièce circulaire minuscule de un mètre vingt de diamètre qui occupe le sommet de cette tour au niveau des combles (fig. 3). La pièce est éclairée par deux petites fenêtres. La charpente 
apparente, supportant un toit de tuiles, tient lieu de plafond (fig. 5) et le sol est recouvert de petits carreaux en terre cuite.

11 Quel a pu être, au cours des temps, l'usage de cette pièce ? Aucun indice, aucune trace de scellement par exemple ne permet de le préciser. Mais elle a dû rester longtemps à l'abandon si l'on en juge par le nombre des graffiti. L'on en trouve du reste dans d'autres pièces de l'immeuble.

12 Dans cette pièce circulaire, le mur de pierre a été, dès l'origine semble-t-i1, enduit d'un mortier de chaux et sable recouvert, en finition, d'un habituel badigeon de chaux non lissé.

$13 \quad \mathrm{Au}$ XVIII ${ }^{\mathrm{e}}$ siècle - ou au tout début du XIX $\mathrm{X}^{\mathrm{e}}$ - une épaisse couche de peinture à l'huile, d'un ton gris-vert assez soutenu, est venue redonner un coup de neuf à la muraille en masquant les graffiti qui la recouvraient et la décrépitude de l'enduit de chaux.

fig. $2 \mathrm{~b}$ : Médaillon représentant Desaix

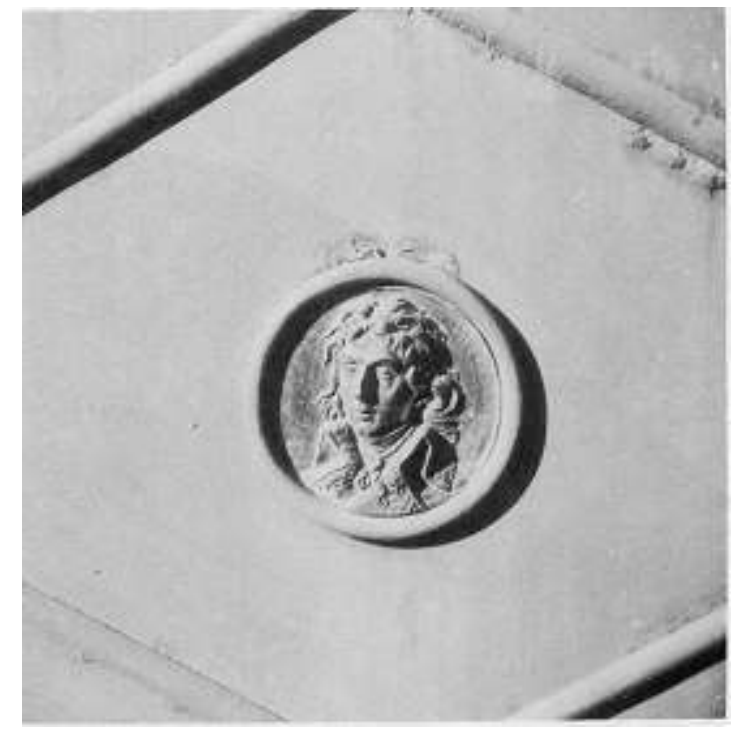

Mirefleurs, quartier du Pavé. Portail de la maison Roubille. De la fonderie, Barbier-Daubrée, 1848.

Photo Ezio Arduini. 
Figure 3 : La tour de la maison de Domat.

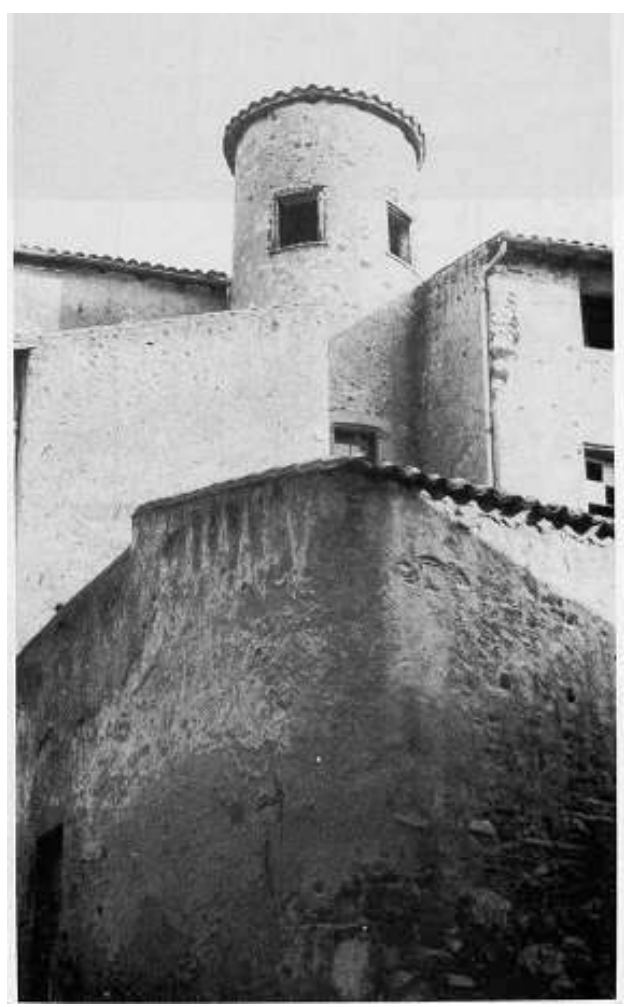

fig. 4 : La maison de Domat.

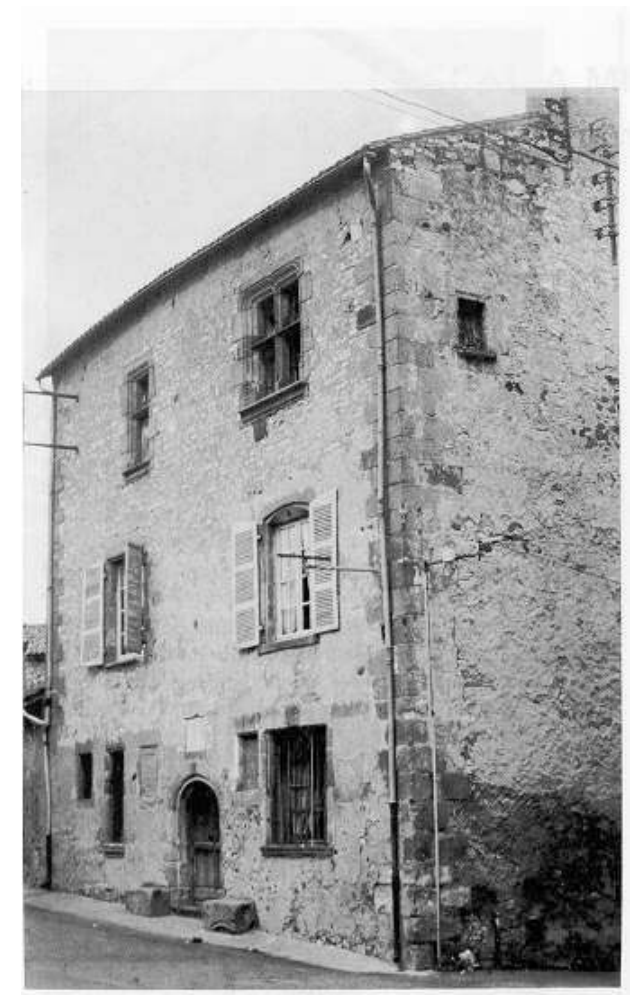

Photo Ezio Arduini. 
Figure 5 : Charpente de la tour.

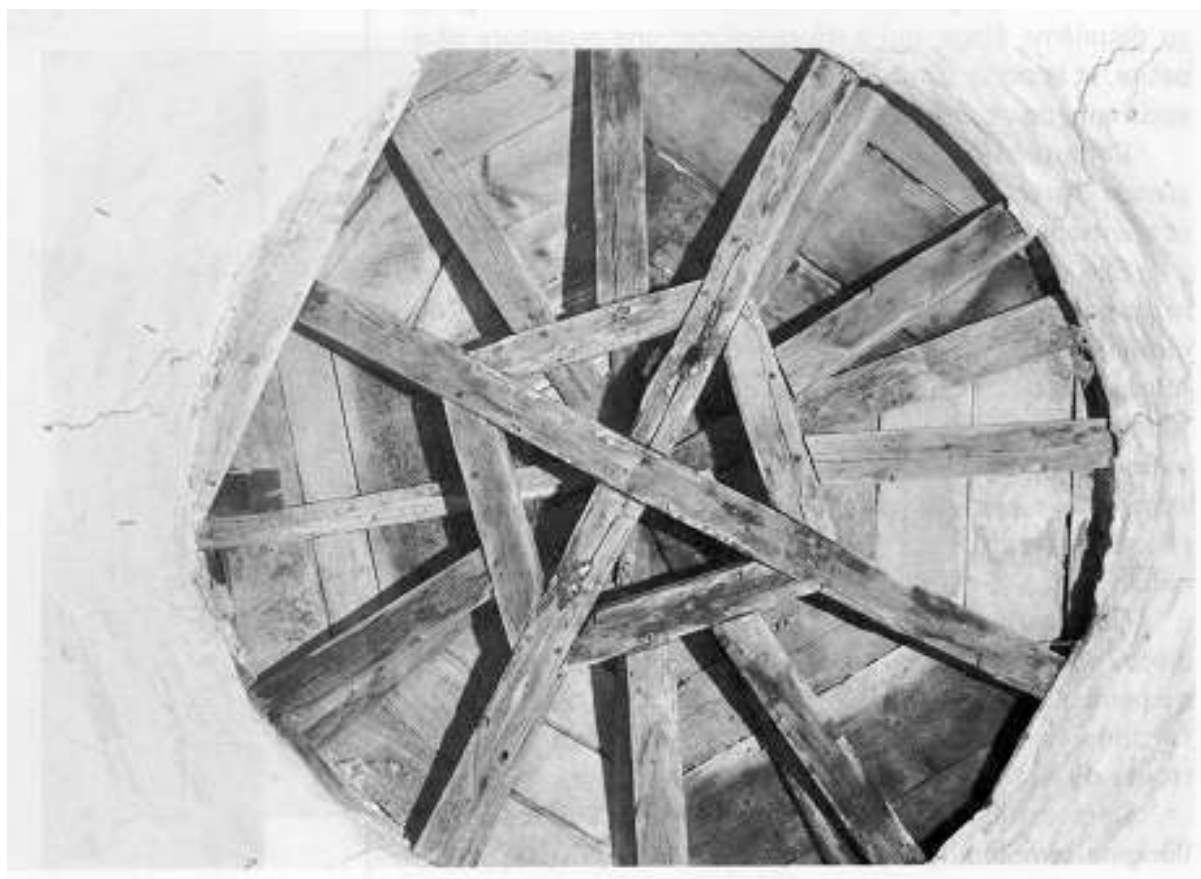

Photo Ezio Arduini.

$14 \mathrm{Au} \mathrm{XIX}^{\mathrm{e}}$ siècle, cette pièce dut être à nouveau occupée car les souillures de la peinture verte étaient encore relativement peu nombreuses quand celle-ci commença, à son tour, à tomber. Grâce à ces décollements de la couche peinte on vit apparaître plusieurs dessins de visages dont l'un d'eux faisait indiscutablement penser à Blaise Pascal.

Dans l'espoir d'autres découvertes, Monsieur Henri Desfeuilles décidait donc, en 1982, de faire éliminer la couche de peinture du XVIII ${ }^{e}$ siècle pour mettre à jour l'ensemble des graffiti de la tour.

Sous la responsabilité des Affaires Culturelles représentées par Monsieur P.- F. Aleil (alors Conservateur des Antiquités et Objets d'Art), nous avons effectué ce travail de dégagement en laissant de côté, les zones haute et basse qui étaient dépourvues de graffiti, et en conservant un fragment de la couche du XVIII ${ }^{e}$ siècle qui portait deux noms de famille. Voir le schéma d'ensemble et son commentaire (fig. 6). 
Fig. 6 Schéma d'ensemble.

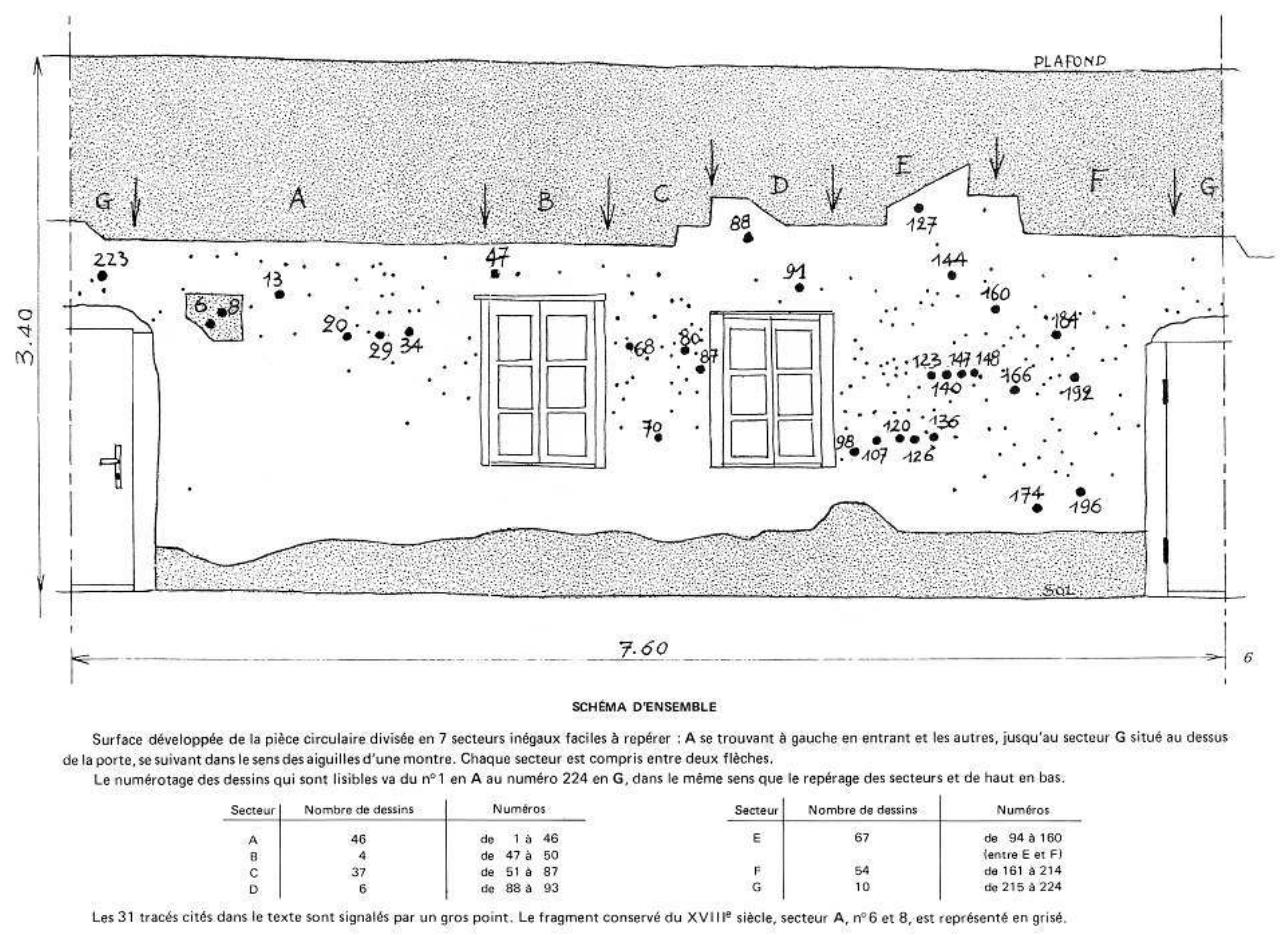

Après avoir consolidé les mortiers affaiblis, comblé les lacunes et nettoyé l'ensemble à la mie de pain, nous avons fixé le pigment de l'ocre rouge à l'aide d'une résine micro poreuse.

\section{Les graffiti. Description générale}

Ces graffiti, au nombre de 225 environ, sont de caractère varié.

Isolés ou enchevêtrés, très affirmés ou à peine lisibles, d'une dimension qui peut aller de quelques centimètres à quelques décimètres, ils sont tracés le plus souvent à l'ocre rouge mais parfois à la mine de plomb. Le trait est la technique le plus fréquemment employée mais les visages sont quelquefois subtilement modelés. Tantôt appliqués et habiles, tantôt sommaires et maladroits, ces graffiti ont de toute évidence, été tracés par des mains différentes au cours d'une période de plusieurs années.

Si certains sont caricaturaux à plaisir, d'autres, très nombreux, paraissent ressemblants. La répétition de certains visages, en effet, montre bien ce souci de la recherche du portrait. (Remarquer les deux visages $n^{\circ} 20$ et 29 et le personnage barbu, $n^{\circ} 80,87,136$, par exemple.)

Ainsi, l'attention du visiteur de la maison de Domat ne peut qu'être attirée tout particulièrement par trois visages qui font immédiatement penser à Blaise Pascal. D'autant plus irrésistiblement qu'on ne visite probablement cette demeure que parce que l'on sait les liens d'amitié qui ont uni Blaise Pascal et Jean Domat.

Mais cette orientation a priori de l'esprit n'exclut nullement que la ressemblance puisse être réelle. C'est ce qu'il nous faut vérifier en toute objectivité. 


\section{Les témoins du visage de Blaise Pascal}

Nous avons choisi de comparer ces trois graffiti au visage de Blaise Pascal pour lequel nous avons retenu deux témoins iconographiques ${ }^{1}$.

24

En premier lieu, le masque mortuaire dont on pense généralement aujourd'hui qu'i1 a servi - par le truchement de contre-moulages en plâtre - de modèle à tous les portraits connus de Blaise Pascal (fig. 7).

Figure 7 a. Masque mortuaire de Pascal. Le contremoulage en plâtre.

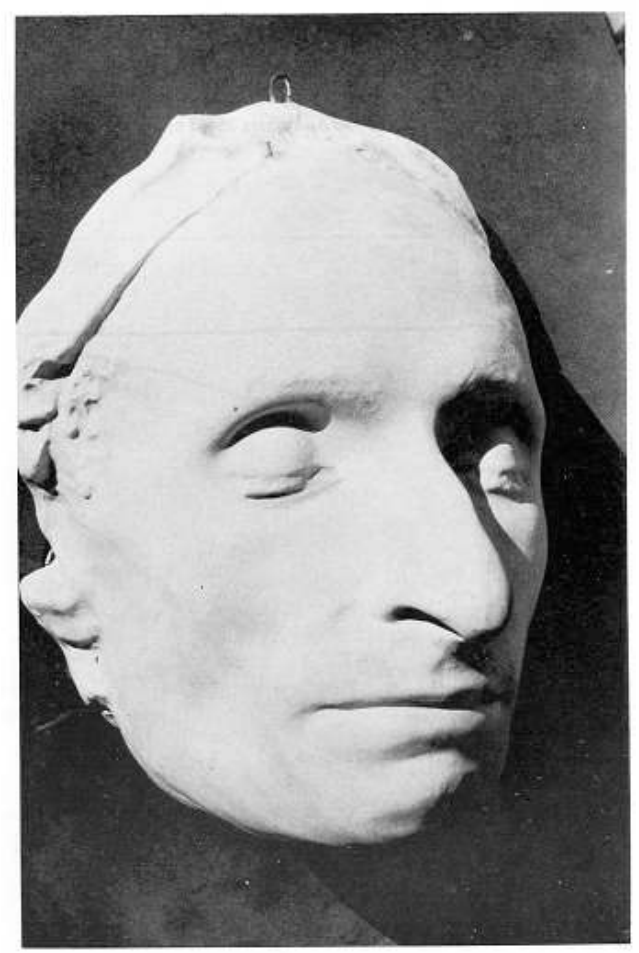

Yves Morvan 
Figure $7 \mathrm{~b}$. Masque mortuaire de Pascal. Le contremoulage en plâtre (de profil)

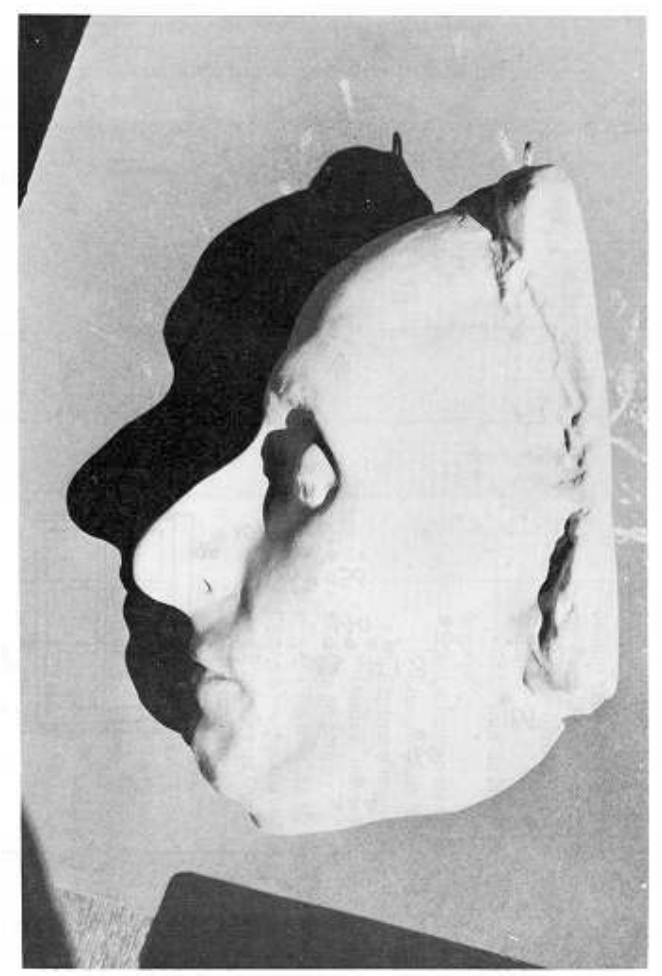

En second lieu, nous avons retenu le portrait à la sanguine, dessiné par Jean Domat, l'ami de Blaise Pascal, représentant ce dernier vers l'âge de seize ans (fig. 8), que nous tenons, jusqu'à preuve indiscutable du contraire, pour fait d'après nature. 


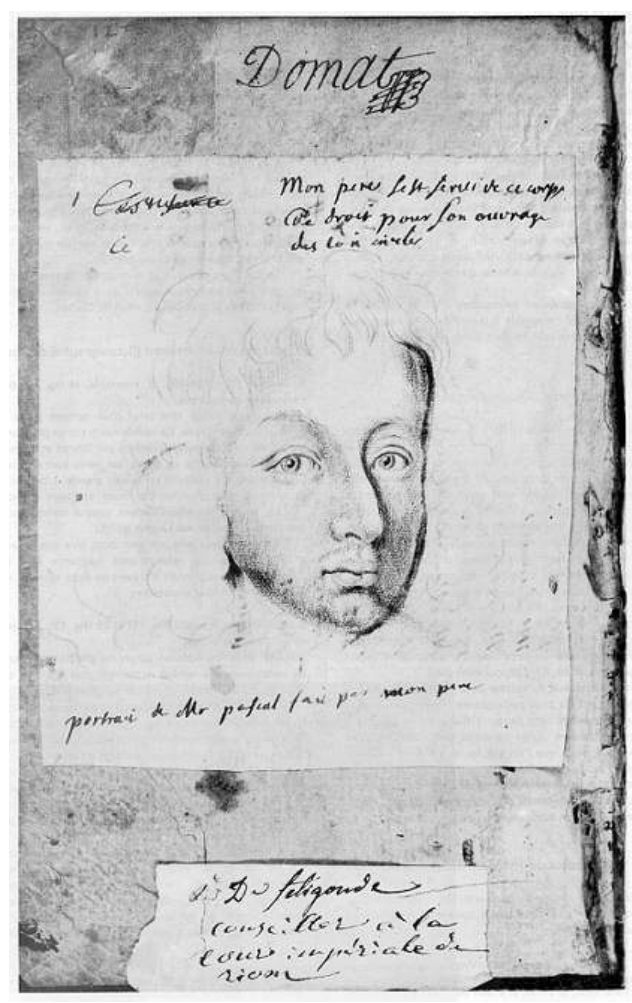

Dessin à la sanguine collé à l'intérieur de la couverture d'un livre de Domat, à la Bibliothèque nationale, Réserve m. F 8 (Voir Blaise Pascal « auvergnat » - Catalogue de l'exposition de Clermont-Ferrand, 1981, $\left.n^{\circ} 115\right)$

Cliché B.N.

Si d'ailleurs le lecteur préfère se référer au portrait posthume attribué par Bernard Dorival à Quesnel II, plutôt qu'au dessin de Domat, la comparaison avec les graffiti de Mirefleurs reste possible puisque ces deux documents présentent les mêmes caractères morphologiques que le masque mortuaire et leur ressemblance avec le modèle n'a été fondamentalement mise en cause par personne.

Le masque mortuaire et le dessin de Domat sont donc les deux seuls témoins auxquels nous allons comparer les trois graffiti de la tour de la maison de Domat.

\section{Trois dessins intéressant l'iconographie de Pascal}

Le visage parait être celui d'un homme jeune entre vingt-cinq et trente ans. De nombreux traits de physionomie rappellent ceux du portrait dessiné par Domat et du masque mortuaire : le menton est court ; les lèvres sont charnues et bien ourlées. La prunelle est plutôt grande. L'œil est rond et enfoncé dans l'orbite. Le front est haut et bombé.

Le nez, s'il est effectivement busqué comme ceux du dessin et du masque, est ici plus pointu. 
31 À cette réserve près, on peut donc dire que ce graffita $n^{\circ} 184$ présente une ressemblance frappante avec Blaise Pascal tel qu'on le connaît à travers les deux témoins iconographiques les moins discutables.

Ces deux exemplaires du même graffita représentent un personnage qui semble nettement plus âgé que le précédent et que Pascal lui-même au moment de sa mort.

Comparée au dessin de Domat et au masque, la bouche en est très différente : la ligne générale est beaucoup plus large, les lèvres sont beaucoup plus minces et les commissures, tirées vers le bas, forment une sorte de pli amer.

Le nez est plus busqué, plus pointu, nettement plus long, plus tombant que celui du dessin ou du masque.

Les yeux ne donnent pas l'impression d'être particulièrement enfoncés dans l'orbite et le front parait être plutôt fuyant que bombé.

D'autre part, on observe que la chevelure présente des ondulations et est relativement volumineuse même au sommet du crâne. De chaque côté du visage cette chevelure tombante creuse une ombre profonde entre elle et la joue droite. Ce type de chevelure correspond bien, jusqu'ici, à celle du visage de Pascal jeune dessiné sur papier par Domat (notre fig. 8) À l'épaisseur près cependant de la masse chevelue du graffito qui semble nettement plus importante au sommet du crâne que celle observée par Domat.

Figure 9 Secteur F, ensemble. $n^{\circ} 184$ Personnage vu de profil.

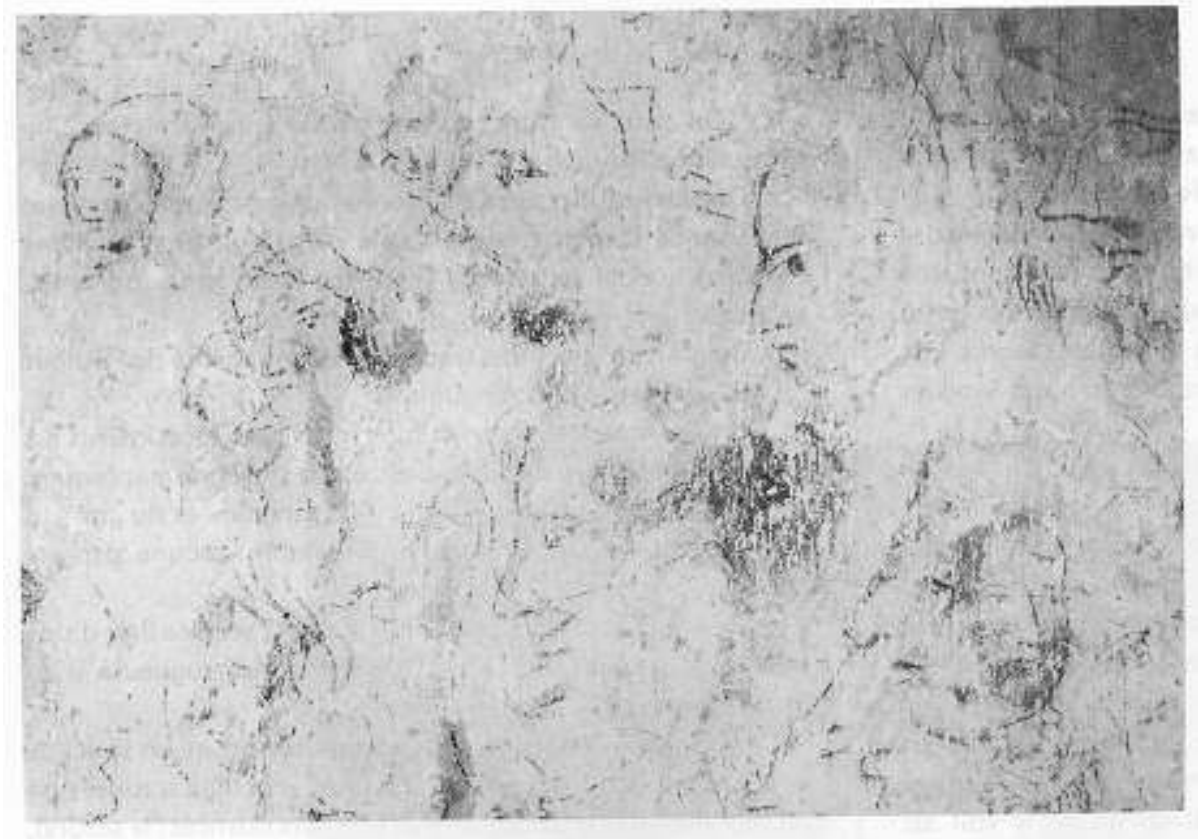


fig. 10 : Secteur F, $n^{\circ} 184$ Personnage vu de profil, détail.

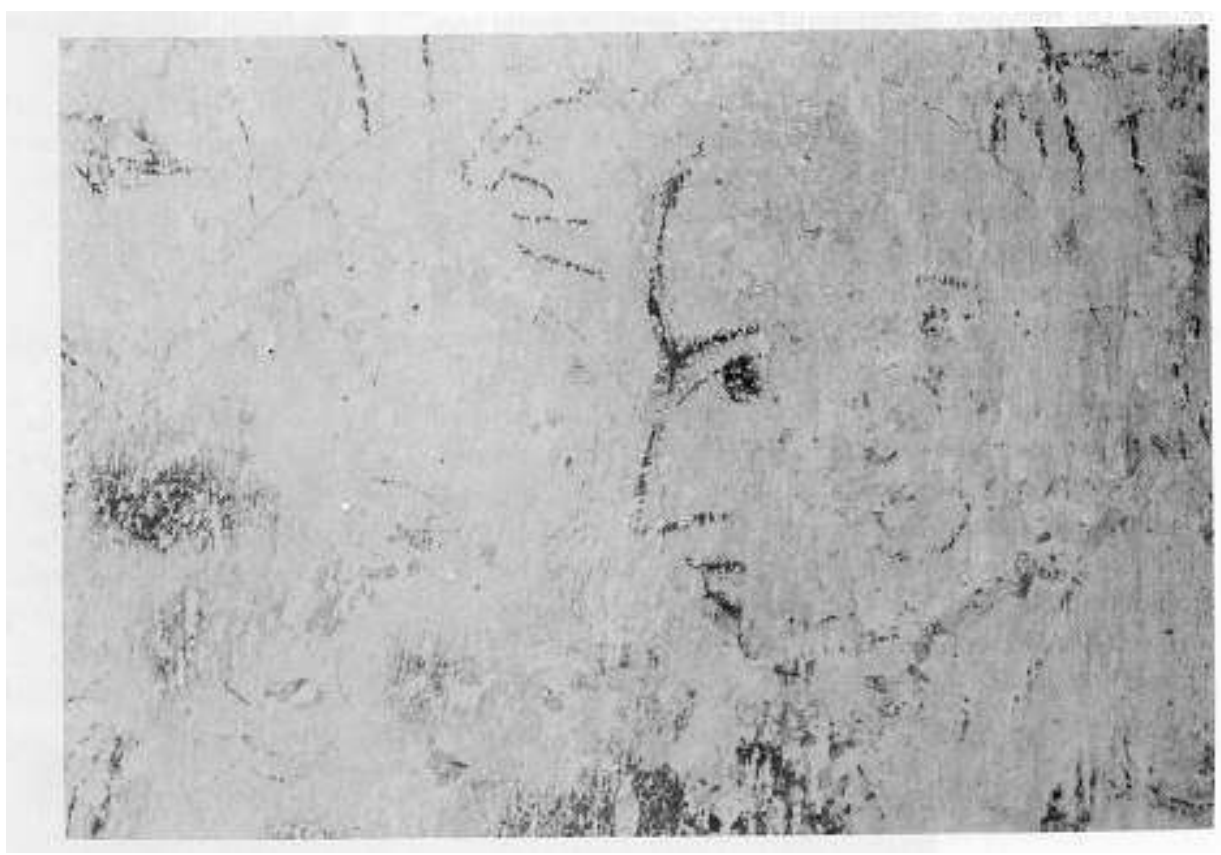

Figure 11 : Secteur $A$, dessin $n^{\circ} 20$ Personnage vu de trois quarts face.

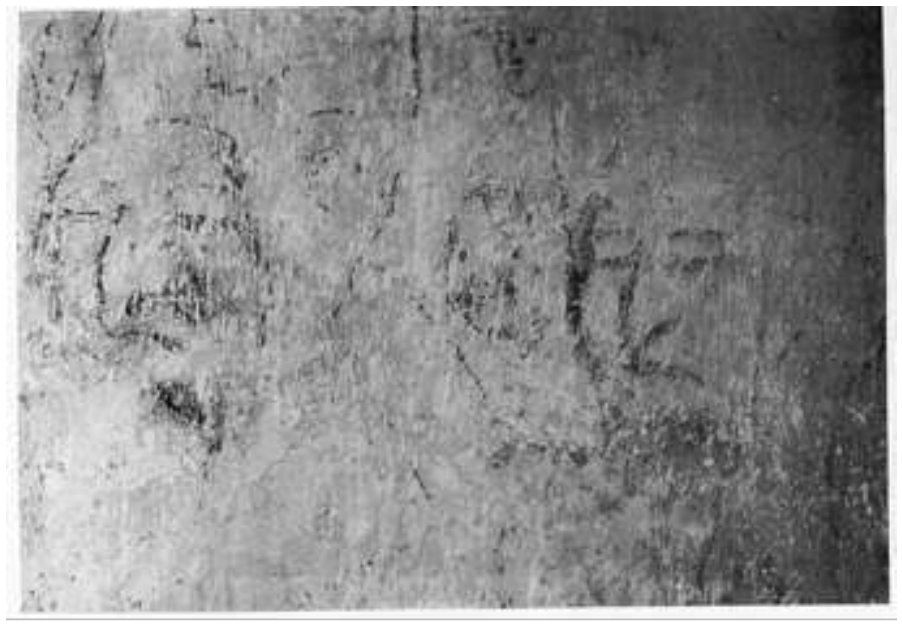


Figure 12 : Dessin $n^{\circ} 29$. Personnage vu de trois-quarts face.

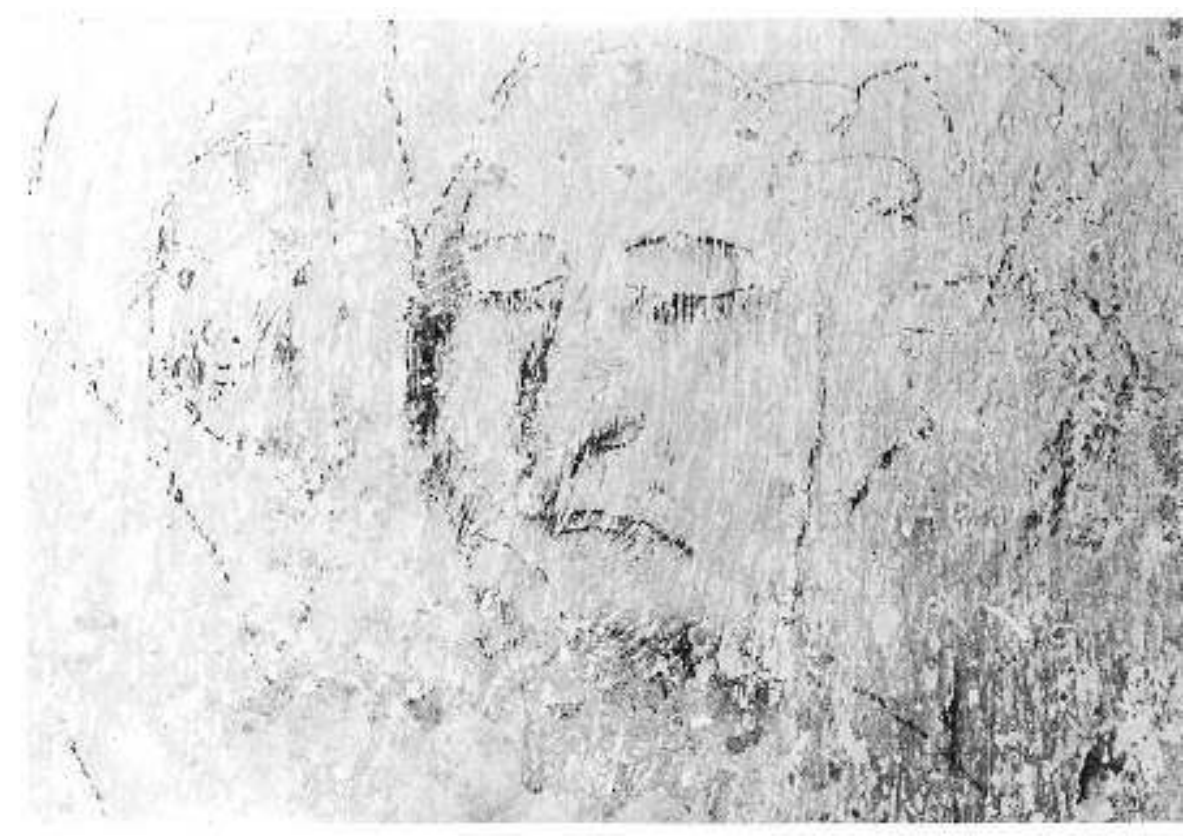

Mais une autre différence, plus évidente, éloigne encore ces deux visages. Les deux esquisses mura les montrent une implantation capillaire frontale qui suit une ligne très nette et indique que les cheveux sont tirés en arrière, tandis que le dessin sur papier de Domat montre une frange à longues mèches retombant sur le front.

Ces deux esquisses d'un même visage d'homme âgé ne résistent donc pas à une comparaison avec les documents témoins. Si elles font indiscutablement penser à un masque mortuaire (et dans le contexte de la maison de Domat, au masque mortuaire de Pascal), c'est essentiellement en raison des traits émaciés du personnage, de la bouche pincée et des paupières mi-closes.

Tout bien considéré, ce visage ressemble finalement davantage à celui de Jean Domat luimême, tel que l'a gravé François d'après Nicolas Coypel, ou tel qu'on le voit au Musée Du Ranquet à Clermont-Ferrand dans un pastel anonyme authentifié par une attestation de son fils (fig. 13). 
Figure 13 : Portrait de Jean Domat.

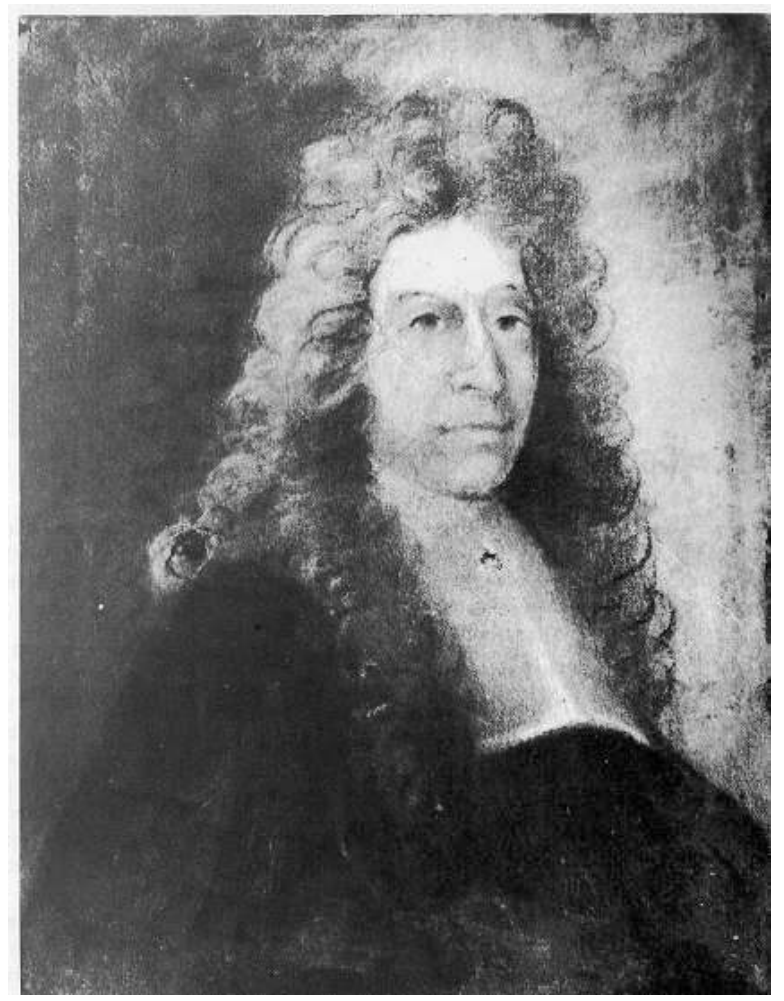

Pastel anonyme. Musée d'art de Clermont-Ferrand. (Voir Blaise Pascal « auvergnat », n 81 et pl. XII)

Cliché C.R.D.P.

En conclusion, seul le personnage $\mathrm{n}^{\circ} 184$, vu de profil, reste un portrait possible de Blaise Pascal. C'est en tout cas celui qui présente avec le dessin sur papier de Domat et le masque mortuaire, des ressemblances.

Ce fait seul, cependant, ne permet pas d'affirmer qu'i1 s'agit d'un portrait de Blaise Pascal. Mais un autre fait vient augmenter cette probabilité. Entre mai 1649 et novembre 1650, Blaise Pascal, son père et sa sœur Jacqueline, fuyant les troubles de la Fronde à Paris, ont vécu à Clermont au foyer de la sœur aînée Gilberte, $\mathrm{M}^{\mathrm{me}}$ Périer. Pascal en profita pour réaliser de nouvelles expériences sur le Puy-de-Dôme et sa liaison avec l'avocat Jean Domat était alors familière. Leur rencontre chez Monsieur de Ribeyre, Premier Président à la Cour des Aides est attestée. Mirefleurs se trouvait à environ une heure et demie d'attelage de Clermont. Domat s'y rendait probablement souvent à la belle saison, en famille - il s'était marié en 1647 - et avec quelques amis. On peut raisonnablement penser que l'ami Pasca1 y fut quelquefois invité.

Dès lors, qui pourrait s'étonner de retrouver, parmi les familiers de Domat croqués sur le mur de la tour, le visage de Blaise Pascal représenté vers l'âge de vingt-six ou vingt-sept ans?

Ce graffito a-t-il été tracé de la main même de l'auteur du fameux dessin à la sanguine?

Une comparaison stylistique entre deux documents est possible lorsqu'on peut la fonder sur des analogies graphiques. Or, le rapprochement du dessin à la sanguine et du graffita de Mirefleurs ne nous a permis d'établir aucune parenté stylistique évidente.

Courrier du Centre international Blaise Pascal, 6 | 1984 
- En $\mathrm{A}, \mathrm{n}^{\circ} 6$, tracé à la mine de plomb sur l'enduit à l'huile d'époque XVIII ${ }^{\mathrm{e}}$ siècle à un endroit que nous avons conservé : « Gilbert Rome » (fig. 14).

Figure 14. $A, n^{\circ} 6$ et 8 . Deux signatures.

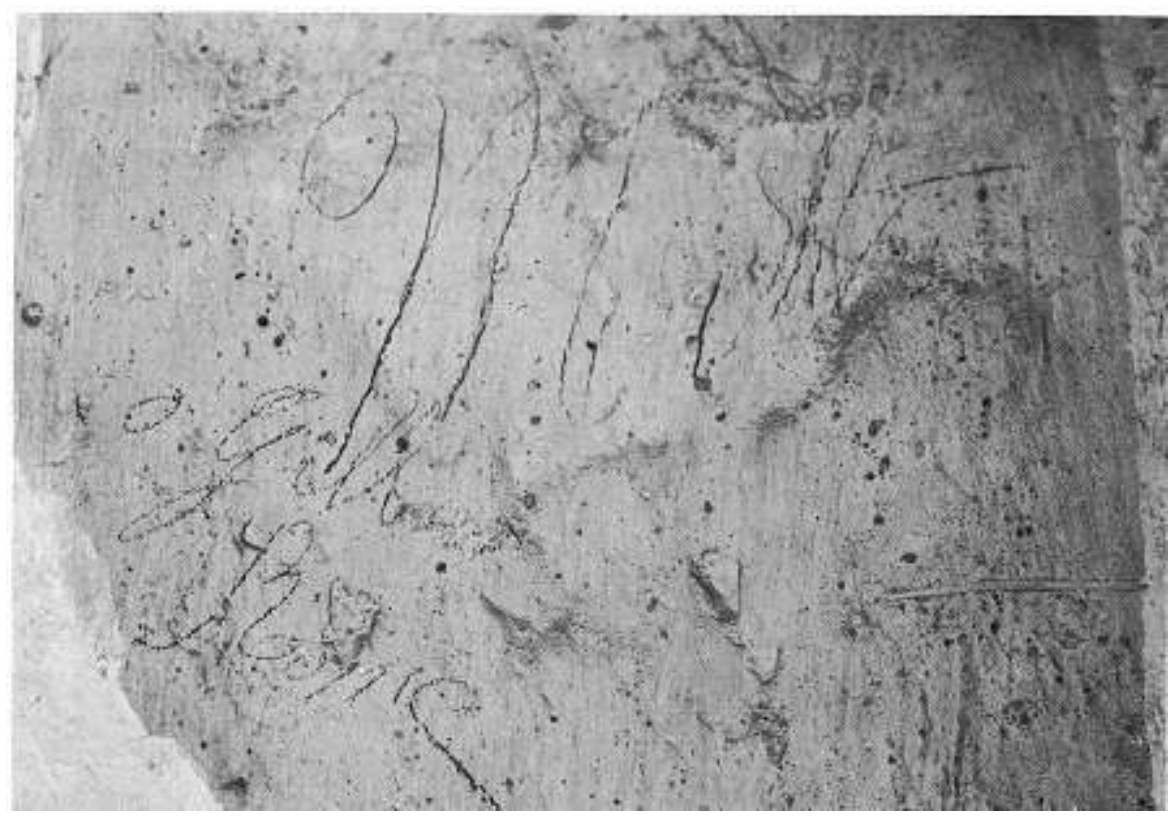

La comparaison avec des signatures du conventionnel riomois conservées aux Archives du Puy-de-Dôme, des différences dans le tracé du $\mathrm{R}$ majuscule et la présence constante de deux $M$ au lieu d'un seul sur le graffito. Sur le mur un signe en forme de 0 précède le nom de Gilbert Rome. M. Bouscayrol nous signale que le patronyme Rome ou Romme était fréquent en Auvergne aux XVIII ${ }^{\mathrm{e}}$ et XIX ${ }^{\mathrm{e}}$ siècle et que le conventionnel «n'est jamais allé à Mirefleurs ». Il doit s'agir d'un homonyme.

- En $\mathrm{A}, \mathrm{n}^{\circ} 8$, « Mitton » (même figure), tracé à la mine de plomb sur l'enduit XVIII ${ }^{\mathrm{e}}$ siècle. M. Bouscayrol découvre dans les ouvrages d'Ambroise Tardieu, "Antoine Mitton, bourgeois, parrain d'Antoine Delarbre à Clermont le 15 janvier 1724. On sait d'autre part que Pascal, pendant sa période "mondaine», en 1653, était en relation avec un "esprit fort » nommé Mitton, Miton ou Myton. Aucun rapport ne peut être établi entre ces deux homonymes, étant donné l'âge de la couche de peinture portant cette inscription.

51 - En D, n 91 (et 88). À la mine de plomb sur badigeon de chaux. En 88 on remarque une grande caricature de profil que l'on retrouve en 91, surchargée de l'inscription suivante :

52 «Mademoiselle Janete Domat. La camuse ». (fig. 15). Voir aussi au n 166 - F. 
Figure 15. $D, n^{\circ} 91$. Caricature et inscription.

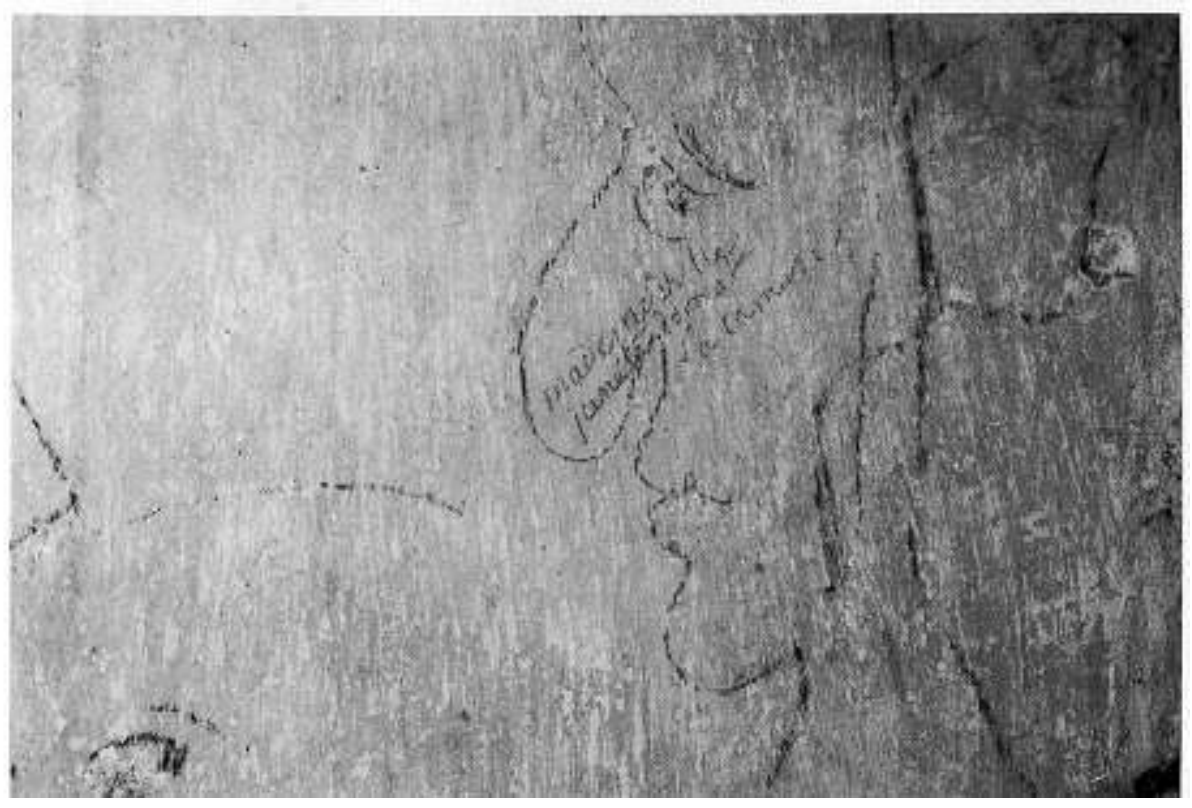

53 - En E, n 127. Mine de plomb sur badigeon ancien. «Lacombe, 173[?]» (l'année serait 1731 ou 1737. Encadré de deux étoiles.)

- En F, $n^{\circ} 166$. Mine de plomb sur badigeon ancien (fig. 16). « Fournet Camu - Chanoine de Saint Pierre. 176[?]» Même écriture qu'au $n^{\circ} 91$. La relation évidente entre les deux inscriptions signifie-t-elle la parenté ou une perfide dénonciation?

Figure 16.F, $\mathrm{n}^{\circ} 166$. Inscription sur dessins.

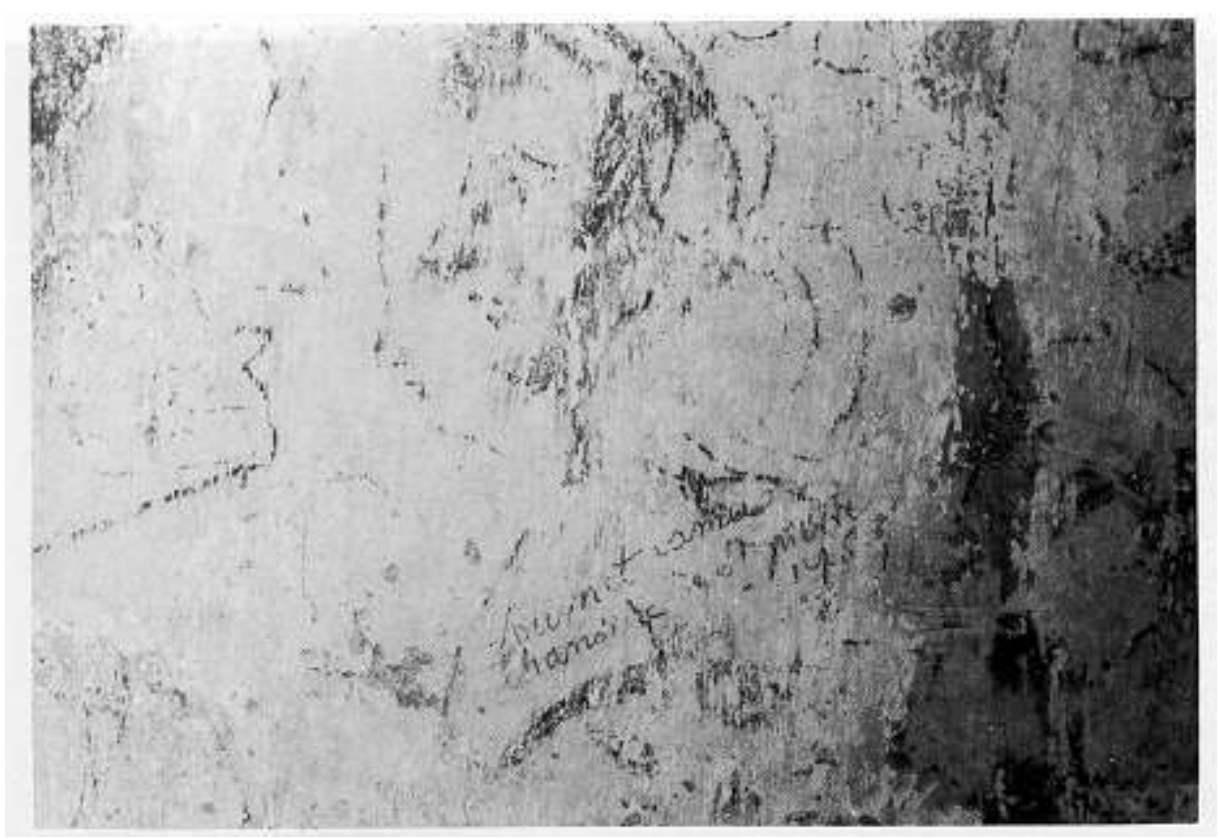

Nous signalons enfin à l'attention du visiteur quelques autres sujets particuliers :

- Anges : têtes d'enfant ailées en : B. 47 ; C. 70 ; E. 123, 140, 147, 148. 
57 - Personnages religieux en : C. 68 : tête d'ecclésiastique, col à rabats; E. 98 : silhouette d'évêque mitré, en pied; E. 107, 120, 126 : religieuses avec coiffe à cornettes (Sœurs de Saint-Vincent de Paul ?)

58 - Trois têtes du même personnage barbu en : C.80, 87 ; E.136.

59 - Vues de bâtiments en : E. 144 : un château à trois tours ; E. 160 : un village entier ; F. 174 et 196 : deux tours esquissées.

60 - Divers : A. 13 : personnage fumant la pipe; A. 34 : un arbre ; F. 192 : personnage assis vêtu à l'antique ; G. 223 : tête de cheval.

\section{NOTES}

1. Nous renvoyons à : Bernard DORIVAL, "L'iconographie de Pascal au XVII ${ }^{e}$ siècle ", in Pascal et Port-Royal, librairie Arthème Fayard, Paris, 1962, p. 91-98, et à Jean MESNARD, «L'iconographie pascalienne», in Pascal, CEuvres complètes, Desclée de Brouwer, 1964, t. I, p.399-419, avec bibliographie.

\section{INDEX}

Mots-clés : Pascal, représentation, Domat (Jean)

Index géographique : Mirefleurs 